\title{
Urinary Tract Infection in Adults
}

\section{Edmond Puca*}

Infectolog, UHC Mother Teresa, Tirana, Albania

*Corresponding author: Edmond Puca, Service of Infectious Diseases, University Hospital Center "Mother Teresa", Tirana, Albania, Tel: 0672058624; E-mail: edmond_puca@yahoo.com

Rec date: Nov 26, 2014; Acc date: Nov 30, 2014; Pub date: Dec 11, 2014

Copyright: (c) 2014 Puca E, et al. This is an open-access article distributed under the terms of the Creative Commons Attribution License, which permits unrestricted use, distribution, and reproduction in any medium, provided the original author and source are credited.

\section{Editorial}

Urinary tract infections (UTIs) are a frequent problem worldwide which are caused by microbial invasion to different tissues of the urinary tract. Urine is normally sterile, that is, free of bacteria, viruses, and fungi. A urinary tract infection is a condition in which one or more parts of the urinary system (the kidneys, ureters, bladder, and urethra) become infected. UTIs are one of the most common bacterial infections in the general population, with an estimated overall incidence rate of 18 per 1000 person per year. It is the most frequent bacterial infection recorded in older people [1]. In addition, UTIs are a major cause of hospital admissions and are associated with significant morbidity and mortality as well as a high economic burden [2]. In a study performed by Sammon et al. 10.8 million patients in the United States visited an Emergency Department (ED) for the treatment of a UTI between 2006 and 2009. The economic burden of utilizing the ED for the treatment of UTIs is estimated to be \$2 billion US dollars annually [3]. UTIs can manifest in a wide clinical range from bacteriuria with limited clinical symptoms to sepsis [4].

1. Depending on the factors that trigger the infections UTIs are classified as:

2. Uncomplicated or complicated,

3. Depending on whether the infection is occurring they are classified as:

4. Primary or recurrent,

5. Depending on sing and symptoms they are classified as:

6. Symptomatic or asymptomatic

A complicated urinary tract infection (cUTI) is an infection associated with a condition, such as a structural or functional abnormality of the genitourinary tract, or the presence of an underlying disease that interferes with host defense mechanisms, which increase the risks of acquiring infection or of failing therapy [1-8]. The primary risk factors for the development of UTI include: age, presence of catheter, chronic comorbidities, neurogenic bladder, diminished mental status, urinary incontinence, diabetes, being female, gynecological disorders, male prostatic hypertrophy ect. Secondary risk factors include dehydration, immobility, other infection, colonization with resistant organisms, and poor personal hygiene. Older adults, especially women, are at increased risk of a secondary infection after the development of a urinary tract infection [2]. The prevalence of UTIs increases in the female population. Pregnancy is one of the factors which increase the risk of UTI partly due to the pressure of gravid uterus on the ureters causing stasis of urine flow and is also attributed to the humoral and immunological changes during normal pregnancy [4]. Estrogen deficiency has been recognized as a risk factor for recurrent UTIs in postmenopausal women because of ensuing vaginal flora changes: protective lactobacilli are replaced by E.coli and other uropathogens [2]. People with indwelling catheters can also be more prone to infections of the bloodstream and they are more generally at risk of urinary infections. [3-5]. The patients are affected by microorganisms capable of inducing inflammation within the urinary and male genital tract. Nearly $95 \%$ of cases of UTIs are caused by bacteria that typically multiply at the opening of the urethra and travel up to the bladder. Organisms causing UTI are derived primarily from the aerobic members of the fecal flora. An overwhelming majority of uncomplicated urinary tract infections [95\%] are caused by a single organism. In contrast, infections among hospitalized patients, patients with urinary catheters, or individuals with structural abnormalities of the urinary tract may be polymicrobial. In uncomplicated UTIs Escherichia coli is the leading organism, whereas in complicated UTIs the bacterial spectrum is much broader including Gram-negative and Gram-positive and often multiresistant organisms. The primary goal of managing UTIs is optimal administration of appropriate antimicrobial agent and correction of any underlying genitourinary abnormalities. A rapid diagnosis is critical to meet the requirements of early goal directed therapy [4]. The diagnosis of UTI is particularly difficult in elderly patients, who are more likely to have asymptomatic bacteriuria as they get older. Urinalysis usually provides enough information to start or not treatment. A urine culture can help identify the specific bacteria causing the infection, and determine which type of antibiotics to use.

Treatment of urinary-tract infection is based on its location and on patient characteristics. A variety of antibiotics are available, and choices depend on many factors, including whether the infection is complicated or uncomplicated, primary or recurrent, symptomatic or asymptomatic. Although antibiotics are the first treatment choice for urinary tract infections, antibiotic-resistant strains of E. coli, the most common cause of UTIs, are increasing worldwide.

Depending on difficulties of treatment: recurrent, complicated and upper UTIs are more problematic compare with other types of UTI. Complicated UTI is a very heterogeneous entity, with a common pattern of the following complicating factors:

1. Anatomical, structural or functional alterations of the urinary tract.

2. Impaired renal function, by parenchymal diseases, or pre,-intra,or post renal nephropathies

3. Accompanying diseases, that impair the patient's immune status

The therapy of uncomplicated UTIs is almost exclusively antibacterial, whereas in complicated UTIs the complicating factors have to be treated as well [4]. Whereas community acquired UTIs are often uncomplicated, almost all nosocomial UTIs are complicated infections [1]. Until recently antimicrobial resistance and healthcare associated infections are increasing.

Many studies have indicated that cranberry juice may help decrease the number of symptomatic UTIs, especially for women with recurrent 
urinary tract infections. Cranberries, blueberries, and lignonberry, are three fruits that appear to have protective properties against urinary tract infections. Probiotics are beneficial microorganisms that may protect against infections in the genital and urinary tracts. The bestknown probiotics are the lactobacilli strains, such as acidophilus, which is found in yogurt and other fermented milk products [kefir], as well as in dietary supplement capsules $[9,10]$. Because of the uncertainty regarding the importance of the adaptive immune response in preventing UTI the role of vaccination has been unclear.

However some studies evidenced the undoubted efficacy of vaccines and estrogen, especially in patients with recurrent infection and elderly respectively $[2,11,12]$.

As there are many different causes of underlying abnormality, a simple recommendation cannot be made for the therapy duration. Most clinical trials have evaluated 5-10 days of treatment. Successful antimicrobial therapy will usually ameliorate symptoms promptly, with substantial clinical improvement in 48 to 72 hours. Patients who fail to respond in this time frame should be reassessed to exclude urinary obstruction or abscess [which may require drainage], to exclude resistance of the infecting organism to the antimicrobial agents, or to consider an alternate diagnosis other than urinary infection. Follow-up cultures should be done 2-4 weeks after cessation of therapy to confirm cure [7]. Suppressive antimicrobial therapy may be considered for selected patients with frequent, recurrent, symptomatic infection in whom the underlying genitourinary abnormality cannot be corrected [9]. Guidelines suggest that antibiotic prophylaxis should not be used to prevent catheter associated UTI in catheterized patients. Although prophylaxis may decrease the incidence of asymptomatic bacteriuria in catheterized patients, it increases the risk of antimicrobial resistance.

\section{References}

1. Laupland KB, Ross T, Pitout JD, Church DL, Gregson DB (2007) Community-onset urinary tract infections: a population-based assessment. Infection 35:150-153.
2. Smith AC, Almond M (2007) Management of urinary tract infections in the elderlyTrends in Urology Gynaecology \& Sexual Health 12: 31-34.

3. Sammon JD, Sharma P, Rahbar H, Roghmann F, Ghani KR, et al. (2013) Predictors of admission in patients presenting to the emergency department with urinary tract infection. World J. Urol 32: 813-819.

4. Tazebew E, Getenet B, Wondewosen T, Silabat M (2013) Associated risk factors of urinary tract infection among pregnant women at Felege Hiwot Referral Hospital, Bahir Dar, North West Ethiopia BMC Research Notes 6: 292.

5. Rivers E, Nguyen B, Havstad S, Ressler J, Muzzin A, et al (2001) Early goal-directed therapy in the treatment of severe sepsis and septic shock. N Engl J Med 345: 1368-1377.

6. Wagenlehner FME, Naber KG (2006) Treatment of Bacterial Urinary Tract Infections: Presence and Future. European urology 49: 235-244

7. Kumar A, Roberts D, Wood KE, Light B, Parrillo JE, et al (2006) Duration of hypotension before initiation of effective antimicrobial therapy is the critical determinant of survival in human septic shock. Crit Care Med 34: 1589-1596.

8. Kalra OP, Raizada A (2009) Approach to a Patient with Urosepsis. J Glob Infect Dis. 1: 57-63.

9. Pallett A, Hand K (2010) Complicated urinary tract infections: practical solutions for the treatment of multiresistant Gram-negative bacteria. J Antimicrob Chemother 65 Suppl 3: iii25-33.

10. Nicolle LE (2001) A practical guide to antimicrobial management of complicated urinary tract infection. Drugs Aging 18: 243-254.

11. Gómez MFL, Fernández BP, García-Criado FJ, Canelo JAM, Vicente AG, et al. (2013) Evaluation of a therapeutic vaccine for the prevention of recurrent urinary tract infections versus prophylactic treatment with antibiotics. Int Urogynecol J 24: 127-134

12. Kucheria R, Dasgupta P, Sacks SH, Khan MS, Sheerin NS (2005) Urinary tract infections: new insights into a common problem. Postgrad Med J 81: 83-86. 